\title{
Abkehr von Schönheit und Ideal in der Liebeslyrik
}

\author{
Symposion an der Humboldt-Universität zu Berlin \\ aus Anlaß des 65. Geburtstages von Peter Brockmeier \\ 8.-11. April 1999
}

Das dem Romanisten und Komparatisten Peter Brockmeier zum 65. Geburtstag gewidmete Symposion Abkehr von Schönheit und Ideal in der Liebeslyrik wurde am 8. April mit einem feierlichen Festakt im Senatssaal der HumboldtUniversität durch die Vizepräsidentin der Humboldt-Universität, Prof. Dr. Ursula Schaefer, eröffnet. Nach Grußworten von Prof. Dr. Pierangelo Schiera (Istituto Italiano di Cultura) und Dr. Hubert Guicharrousse (Institut Français) wurden die drei ersten von insgesamt 22 Vorträgen gehalten. Es folgte ein feierlicher Festakt, der vom Dekan der Philosophischen Fakultät II, Prof. Dr. Helmut Pfeiffer, eröffnet und durch zwei Beiträge von Prof. Dr. Stéphane Michaud (Paris III-Sorbonne Nouvelle) und Dr. Bernd Lutz (Metzler Verlag) - mit beiden verbindet Peter Brockmeier eine langjährige Zusammenarbeit - ergänzt wurde. Ein anspruchsvolles musikalisches Rahmenprogramm (Gesang: Susanne Heckel M.A., Klavier: Dr. Bettina Cenerelli) rundete den Eröffnungstag ab.

Der Wahl des Themas „Abkehr von Schönheit und Ideal in der Liebeslyrik“ lag die Beobachtung zugrunde, daß sich zwar eine Vielzahl von Publikationen mit Aspekten der Liebeslyrik oder der Ästhetisierung des Häßlichen beschäftigen, jedoch nur selten der Versuch unternommen wird, diese unter einer übergeordneten Fragestellung miteinander zu verbinden. Erklärtes Ziel der Veranstalterinnen Carolin Fischer und Carola Veit war die Relativierung der Vorstellung, daß Liebeslyrik ausschließlich von der Idealisierung einer Geliebten geprägt sein müsse. Vielmehr habe es neben der idealisierenden Liebeslyrik stets contre-textes gegeben, die vorgegebene Muster parodierten oder auf andere Weise umbildeten. Das Thema sollte aus einer komparatistischen Perspektive diachron bearbeitet werden, wobei gattungshistorisch und -poetologisch sowie thematisch-inhaltlich ein offener Begriff von Liebeslyrik vorausgesetzt wurde.

Entsprechend weit - vom Mittelalter bis zum 20. Jahrhundert - war der zeitliche Rahmen gesteckt. Angelica Rieger (Wiesbaden) vertrat in ihrem Vortrag die These, daß die Lyrik der Trobadors eben nicht nur der höfischen Konvention Genüge tat, sondern diese auch mit obszönen Anspielungen und sehr direkten Aufforderungen konterkarierte. Zeitlich noch weiter zurück ging Ursula Schäfer (HU Berlin) mit einer Untersuchung der altenglischen Liebesgedichte The Wife's Lament und Wulf and Eadwacer. In ihnen beklagt jeweils eine weib- 
liche persona die Trennung von einem Mann, wobei gerade die unterschiedlichen Deutungen dieser Klage die Problematik des Codes „Liebe“ aufzeigen können. Abgeschlossen wurde der Komplex zum Mittelalter mit Sebastian Neuschäfer (FU Berlin), der am Beispiel des katalanischen Dichters Ausiàs March erläuterte, wie in der Gattung des altprovenzalischen planh bereits im 15. Jahrhundert der ,Absolutheit der Liebe [...] pointiert die Relativität des Subjekts entgegengestellt" wird: der Dichter kommentiert innertextlich die eigene Unfähigkeit, sich nach dem Tod seiner Dame das Leben zu nehmen.

Zur Frühen Neuzeit leiteten dann drei anglistische Vorträge über: Theo Stemmler (Mannheim) zeigte, wie Thomas Wyatt in seinen Petrarca-Umdichtungen ,die Frau aus dem Himmel transzendentaler Verklärung auf den Boden erotischer Tatsachen" zurückholte. Gegen den petrarkistischen Prätext hatte auch Shakespeare mit seinen Dark Lady-Sonnets gewirkt, die Alfred Behrmann (FU Berlin) in seinem in englischer Sprache vorgetragenen Beitrag untersuchte. Die Dark Lady, von der es heißt „her brests are dun“, wobei „,dun“ als „dull greyish brown, like the hair of a mouse" zu verstehen ist, dürfte kaum dem höfischen Idealbild, einer Laura oder einer Beatrice, entsprochen haben und auch charakterlich weist sie erhebliche Defizite auf - und doch wird sie, trotzdem oder vielleicht gerade deshalb, geliebt. Auch Martin Brunkhorst (Potsdam) nahm Shakespeare als Beispiel. Er belegte dessen zwiespältige Einstellung zu Petrarca und zur Petrarca-Rezeption anhand der in As you like it und in Hamlet eingefügten Liebesgedichte und dem dort greifbaren Spott auf das höfische Ideal.

Elvio Guagnini (Triest) zeigte, wie sich Francesco Berni, Begrïnder der poesia bernesca, in die Tradition der burlesken und satirischen Dichtung einschreibt und gleichzeitig eine in seinem Verständnis der Rolle des Dichters begründete Gegenposition zur ,klassizistischen' Dichtung der Zeit und zum Petrarkismus einnimmt. Es folgten zwei hispanistische Beiträge: Dietrich Briesemeister (Berlin/Jena) legte am Beispiel Garcilasos de la Vega und damit einem der bedeutendsten frühneuzeitlichen spanischen Autoren von Liebesdichtung dar, wie sich auf der Grundlage zeitgenössischer Melancholie-Vorstellungen „ein Szenarium der Verstörung und des Schreckens“ fassen läßt. Eberhard Geisler (Mainz) zeigte am Beispiel vier berühmter Liebesgedichte Quevedos nicht nur deren unterschiedliche Deutungen, sondern auch die Vielfalt der literarischen Register dieses Autors auf -- vom hohen Liebesdiskurs über dessen Infragestellung bis hin zu seiner satirischen und burlesken Umformung. Wie in der französischen Literatur des 17. Jahrhunderts nicht nur die Liebeslyrik selbst, sondern auch der sich darum rankende Literaturbetrieb verspottet wurde, 
indem verschiedene Autoren Liebesgedichte in Dramen und Romane einarbeiteten und sich so kritisch mit der Gattung auseinandersetzten, erläuterte Wolfgang Theile (Bamberg). Einen epochenübergreifenden Beitrag lieferte Horst Albert Glaser (Essen), der Aspekte der femme machine unter anderem bei Hesiod, Villon und in den Blasons von Marot untersuchte und dabei auf eine allgegenwärtige Fragmentarisierung des weiblichen Körpers stieß, der dann letztlich - wie in Hoffmannswaldaus Gedancken / Uber den Kirchhoff und Ruhestädte der Verstorbenen - doch der Zerstörerin Zeit zum Opfer fällt.

Am folgenden Tag schlug Michel Delon (Paris IV-Sorbonne) die Brücke vom 18. zum 19. Jahrhundert: er wies nach, daß über die Vermittlung Rousseaus und De Sades insofern eine ,conquête de l'imperfection“ stattfand, als nunmehr Häßlichkeit nicht mehr als Gegenpol zu Schönheit zu sehen sei, sondern eine eigene Wertigkeit erlangte. Dem 18. Jahrhundert kommt dabei die Funktion eines Scharniers zu, indem die Dichotomie von schön und häßlich aufgelöst und bewußt übertreten wird. Es folgten zwei germanistische Beiträge, die sich beide Heinrich Heine widmeten. Elena Agazzi (Bergamo) ging auf Heines Nachahmung und Umakzentuierung des petrarkistischen Modells ein, die in dem ironischen Vers gipfelt ,Und wenn meine Liebste ein Herzchen hätt, / ich machte darauf ein hübsches Sonett". Chris Rauseo (FU Berlin) nannte Schuberts Vertonungen von Heine-Gedichten aus dem Buch der Lieder ein Mißverständnis, welches daraus resultiere, daß Heines Gedichte als Abkehr von romantischen Lyrikkonventionen und Schuberts Musik als Rückkehr zu romantischen Musikkonventionen zu interpretieren seien.

Am Nachmittag des Tages wandte man sich dem französischen 19. Jahrhundert und damit den Autoren zu, die als prototypische Vertreter einer Ästhetisierung des Häßlichen gelten. Den Zusammenhang von Kunst und Literatur führte Bettina Cenerelli (Paris) am Beispiel Théophile Gautiers aus. An die Stelle einer konkreten Person tritt ein Kunstwerk, das besungen wird, und so lesen sich Gautiers Gedichte zwar noch wie Liebeslyrik, doch wird in einem Übertragungsprozeß „ein neues dichterisches Kunstwerk" und damit „die Voraussetzung für die ästhetische Kehrtwende, die seine Nachfolger einschlagen werden“ geschaffen. Max Milner (Paris) zog den wichtigsten Autor der Ästhetisierung des Häßlichen heran und zeigte, wie sich Idealisierung und Derbheit bei Baudelaire zu einer Ironie verbinden, die traditionelle Verfahren von Liebeslyrik konterkariert. Auch Wolfgang Drost (Siegen) widmete sich Baudelaire und erläuterte in seiner Lektüre von Une Charogne, wie über den das Gedicht durchwehenden Aasgeruch die Häßlichkeit der Liebe vermittelt und der Bruch mit der poetischen Tradition vollzogen wird. Es folgte der Vortrag von Stéphane Mi- 
chaud (Paris III-Sorbonne Nouvelle), der am Beispiel der literarischen Gestaltung der Madonna bei Rilke darlegte, inwiefern dieser - wenn auch zunächst nur implizit - Baudelaire folgte und sich mit der Ästhetik des Häßlichen auseinandersetzte.

Der letzte Tag des Symposions wurde mit einem Beitrag von Manfred Hinz (Passau) eröffnet: über eine Vergleich von Saba und Weininger verwies er auf den Zwiespalt zwischen dem petrarkistischen Modell und der durch die psychoanalytische Erforschung hoffähig gemachten offen ausgeprochenen Sexualität. Alexandra Beilharz (HU Berlin) ging Melancholie und Entfremdung in Liebesgedichten Luis Cernudas nach: das die Liebeslyrik charakterisierende Streben nach Vollkommenheit wird bei Cernuda in Frage gestellt und - häufig im Rückgriff auf christliche vanitas-Thematik - im Ergebnis resignativ verneint. Jean Bessière (Paris III - Sorbonne Nouvelle) sprach von der Grausamkeit als Spiel mit dem Negativen bei René Char, wobei er Grausamkeit als Dekonstruktion von Traum und amour fou bestimmte. Abgeschlossen wurde dieser Tag mit einem Petrarca ist wieder in Sicht überschriebenen Beitrag des Celan-Forschers Hans-Michael Speier (Berlin/Cincinnati). Er wies unter anderem an Celans Gedicht Lößpuppen eine ,sexuelle Poetik“ im Werk des Autors nach, für den Schreibakt und Liebesakt identisch gesehen werden können. Ergänzt wird der Tagungsband um zwei Aufsätze von Fritz Wagner (FU Berlin, Die derbe Venus in der Vagantendichtung) und Irmgard Osols-Wehden (Mainz, Die entlarvte Geliebte und der entthronte Liebhaber in den burlesken Dichtungen Paul Scarrons) sowie um die Beiträge von den Teilnehmern, die aufgrund ihrer Funktion als Veranstalterinnen beziehungsweise Moderatoren während des Symposions nicht vorgetragen hatten: Carolin Fischer (HU Berlin) zeigt, wie sich über die Gestalt der häßlichen Alten bei Du Bellay und Ronsard die „Schattenseiten der Liebe“ in die idealisierende Dichtung einschleichen. Karl Maurer (Bochum) belegt am Beispiel von Leopardis Ultimo Canto di Saffo, „wie weit sich der Dichter seit seinen Anfängen von der durch Petrarca begründeten Tradition entfernt hat". Hermann H. Wetzel (Regensburg) konstatiert im Hinblick auf „Rimbaud als Verfasser von Liebesgedichten“, daß dieser in allen Etappen immer wieder Desillusionierung und Enttäuschung bezüglich eines weiblichen - Ideals thematisiert. Carola Veit (HU Berlin) schließlich geht auf Gedichte Becketts ein, anhand derer sich eine auch chronologische Entwicklung im Werk des Autors von jugendlichen Ausschweifungen bis hin zur Thematik der Liebe im Alter nachvollziehen läßt, wobei letztere allerdings den körperlichen Verfall in den Szene setzt. 
Als Ergebnis des Symposions kann festgehalten werden, daß keineswegs erst Baudelaire dem Häßlichen Eingang in die Lyrik verschafft, sondern die Abkehr von Schönheit und Ideal viel früher und unabhängig von Subgattungen stattgefunden und somit innerhalb der Liebesdichtung eine eigene Tradition begründet hatte. Eine Tradition, deren Bandbreite weit über die burlesken Umdeutungen des Antipetrarkismus oder die auf grausamen oder ekelerregenden Details basierenden ästhetizistischen Darstellungen des Symbolismus hinausgeht. Ein Resultat der Tagung ist damit auch die Konstatierung eines offenen und somit schillernderen Schönheitsideals der Dichter als das ausschließlich durch den petrarkistischen Liebesdiskurs präformierte. Der Bandbreite des Geehrten, dessen eigene Forschungen von Villon bis Beckett reichen und neben den drei großen romanischen Sprachen auch die englische und deutsche Literatur umfassen, wurde damit in vieler Hinsicht entsprochen. Die Beiträge dieses rundum gelungenen Symposions, das von einer auch über die wissenschaftliche Diskussion hinausgehenden erfreulichen Atmosphäre geprägt war, sind demnächst in der Reihe M\&P des Metzler Verlages nachzulesen: die Publikation ist für Frühjahr 2000 geplant.

Alexandra Beilharz

\section{Das Neue - Eine Denkfigur der Moderne}

\section{Tagung der Deutschen Gesellschaft}

für Allgemeine und Vergleichende Literaturwissenschaft, Internationales Wissenschaftsforum, Heidelberg, 26.-29. Mai 1999

Die XI. Tagung der Deutschen Gesellschaft für Allgemeine und Vergleichende Literaturwissenschaft, die dem Thema Das Neue - Eine Denkfigur der Moderne gewidmet war, fand vom 26. bis 29. Mai im Internationalen Wissenschaftsforum der Universität Heidelberg statt, dessen ansprechender Rahmen nicht unwesentlich zum guten Gelingen der Veranstaltung beitrug.

Als Syndrom par excellence der Neuzeit bietet sich die Denkfigur des Neuen in ihren unterschiedlichen (literarischen, philosophischen und theologischen) Facetten zu einem interdisziplinären Dialog über die konstitutiven Bedingungen der Moderne in besonderer Weise an, begreift diese sich doch in Fiktion eines radikalen Kontinuitätsbruchs als emphatische Neusetzung und Überbietung des vermeintlich Alten. Die grundlegende Bedeutung des Neuen als tradi- 